\title{
Information processing in space-time by the hippocampus
}

\author{
JOHN W. MOORE \\ University of Massachusetts, Amherst, Massachusetts 01003
}

\begin{abstract}
Black, Nadel, and O'Keefe (1977) have proposed that the main function of the hippocampus in behavior is to engage in spatial mapping, i.e., to provide the cognitive maps which form the basis of place strategies. They review evidence that such strategies play a role in certain avoidance and punishment situations and that hippocampal damage affects these strategies while leaving unaffected cue strategies derived from temporal processing. The evidence supporting the spatial mapping hypothesis is examined in terms of an alternative notion, namely, that the hippocampus enables the animal to ignore (tune out) irrelevant stimuli and events. Despite the limitations of such ad hoc analyses, a case is made that deficits in spatial learning might ultimately be traced to (a) persistence of inefficient modes of responding under the control of irrelevant stimuli and (b) failures of associative learning due to interference from irrelevant incongruous events. It is suggested that the hippocampus is part of a general system that processes information in both space and time.
\end{abstract}

Black, Nadel, and O'Keefe (1977) have suggested that the principal function of the hippocampus in behavior is to process information about places. A distinction is made between spatial processing, which they refer to as spatial mapping, and the processing of temporal relationships. Spatial mapping involves the hippocampus as a neural substrate; temporal processing presumably does not. Black et al. (1977) advanced this hypothesis by considering the extensive literature on avoidance learning and punishment in which animals, typically rats, have suffered damage to the hippocampal formation or related structures. Recent studies involving other behavioral paradigms, which will be considered later in this article, provide perhaps even more impressive evidence that the hippocampus is implicated in spatial learning and memory in a fundamental way.

One objection to the spatial mapping view is that it ignores evidence that the hippocampus is also involved in temporal processing. For example, Solomon (1979) argues that Black et al. (1977) overlooked evidence from classical conditioning experiments with rabbits. Some of this evidence suggests an altogether different function of the hippocampus, namely that of enabling the animal to ignore (tune out) irrelevant stimuli. To do this, Solomon (1979)

The first draft of this article was written while the author was on the staff of the MRC Unit on Neural Mechanisms of Behavior, University College London, England, I. S. Russell, Director. I wish to thank the following individuals for their kind criticisms of that draft: J. J. B. Ayres, N. E. Berthier, R. K. Clifton, H. G. Marchant III, and P. R. Solomon. Preparation of the revised draft was supported by NSF Grant BNS-7714871. Reprint requests should be made to John W. Moore, Department of Psychology, Middlesex House, University of Massachusetts, Amherst, Massachusetts 01003. suggests that the hippocampus must participate in "temporal mapping," whereby the animal can relate its behavior to a sequence of events.

The evidence cited by Solomon (1979) indicating the involvement of the hippocampus in processing information about the events in the time domain (temporal mapping) is at least as compelling as the evidence favoring a spatial mapping viewpoint. If the data supporting the spatial mapping hypothesis and the tuning-out hypothesis are valid, it is possible that the two are not mutually exclusive and that the evidence supporting the one might be reconciled with the other.

One of the principal themes of the present article is that deficits in spatial learning demonstrated by animals suffering hippocampal damage may be the result of interference arising from inability to tune out irrelevant events. Two kinds of interference are considered: first, interference resulting from failure to tune out stimuli that elicit inappropriate behavior (interference from incompatible responding); secondly, failure of associative learning resulting from inability to tune out distracting irrelevant events or episodes. A hypothesis as general as this tends to blur the fine edge of the distinction between spatial mapping and temporal processing drawn by Black et al. (1977), and the ultimate usefulness of the distinction as a device for understanding the hippocampus becomes questionable.

\section{Tuning-Out Hypothesis}

Solomon's hypothesis that the hippocampus is involved in tuning out irrelevant stimuli (see Moore, 1979; Solomon, 1977; Solomon \& Moore, 1975) may be contrasted with older views of hippocampal 
function that have stressed the role of the hippocampus in tuning out nonreinforced stimuli (e.g., Douglas, 1972). Reinforcement does not guarantee relevance, nor does nonreinforcement guarantee irrelevance. Irrelevant stimuli are broadly conceived as any which would lead to behaviors that are inefficient, inappropriate, or maladaptive. In this regard, the tuning-out hypothesis resembles that used to characterize certain forms of clinical amnesia, e.g., Weiskrantz and Warrington's (1975) hypothesis that the amnesia observed in Korsakoff's patients derives from an inability to tune out irrelevant memories based on prior experience.

The primary behavioral evidence for Solomon's (1979) version of the tuning-out hypothesis comes from experiments of classical conditioning in rabbits and rats that show deficits in latent inhibition and Kamin blocking in animals with hippocampal damage.

In a latent inhibition experiment, the animal is repeatedly preexposed to the to-be-conditioned stimulus prior to the introduction of reinforcement, i.e., prior to the pairing of the conditioned stimulus with the unconditioned stimulus. Normal animals show evidence of retarded classical conditioning, presumably becuse they have learned to ignore or tune out the conditioned stimulus during the preexposure phase (see Mackintosh, 1975), whereas conditioning in animals with hippocampal damage proceeds as though the conditioned stimulus had never been experienced prior to pairing with the reinforcer (McFarland, Kostas, \& Drew, 1978; Solomon \& Moore, 1975). It should be emphasized that hippocampal damage does not appear to impair the process of acquiring the conditioned response. ${ }^{1}$

In a Kamin blocking experiment, the animal acquires a conditioned response to one conditioned stimulus (A) prior to additional conditioning trials in which a new conditioned stimulus $(\mathrm{X})$ is presented together with stimulus A. In normal, intact animals, subsequent testing of conditioned responding to $\mathrm{X}$ presented alone typically shows that $\mathrm{X}$ is only weakly conditioned in comparison with control animals that had been conditioned to the compound conditioned stimulus (AX), but without the prior conditioning to stimulus $\mathrm{A}$ alone. The fact that stimulus $\mathrm{X}$ is only weakly conditioned in the first instance is the Kamin blocking effect. At least one influential interpretation of this phenomenon is that the prior conditioning to stimulus $\mathrm{A}$ renders stimulus $\mathrm{X}$ a redundant (hence, irrelevant) predictor of the unconditioned stimulus. The animal learns to ignore or tune out stimulus $\mathrm{X}$, and this attentional barrier prevents it from becoming strongly associated with the reinforcing event (Mackintosh, 1975).

Assessments of Kamin blocking in animals with hippocampal damage have indicated strong con- ditioning to stimulus $\mathrm{X}$ during $\mathrm{AX}$-compound conditioning trials despite prior conditioning to stimulus A (Rickert, Bennett, Lane, \& French, 1978; Solomon, 1977). Given an attentional interpretation of Kamin blocking, it would appear that hippocampal animals are less able than normals to tune out the redundant stimulus $\mathrm{X}$, and it therefore becomes strongly conditioned. Since animals with hippocampal damage are prone to learn associative relationships that would normally be blocked by prior learning, such animals would be under the control of irrelevant learned associative relationships that could either act in an agonistic or antagonistic fashion in any given behavioral testing situation.

The foregoing consideration of Kamin blocking illustrates the idea that association with reinforcement does not always guarantee a stimulus's relevance, thereby rendering it immune to tuning out by mechanisms requiring an intact hippocampus. Nor does nonreinforcement guarantee irrelevance and, consequently, tuning out by hippocampal mechanisms. This point has been emphasized by Solomon (1977), who failed to find deficits by hippocampectomized rabbits in Pavlovian conditioned inhibition training. This type of training requires that the animal perform a differentially conditioned response such that it responds to a reinforced stimulus (A) but suppresses responses to a nonreinforced compound stimulus (AX). In order to successfully differentiate between the two types of stimulus configurations, A and $\mathbf{A X}$, the animal must attend to stimulus $\mathrm{X}$. Since tuning out or learning to ignore is not a normal requirement of this task, there are no differences between hippocampally damaged animals and normals. ${ }^{2}$ By contrast, latent inhibition does appear to involve a tuning-out process, and hippocampally damaged animals show the "deficit" of acquiring a conditioned response more quickly than do normal animals that had been similarly preexposed to the conditioned stimulus (Solomon \& Moore, 1975).

To sum up, evidence for the tuning-out viewpoint comes primarily from classical conditioning paradigms, mainly the rabbit eyeblink conditioning. Although analogous findings have been reported in rats, the sheer weight of the potentially relevant literature involving rats with hippocampal-related lesions would suggest that the tuning-out hypothesis, like any single hypothesis, must fail to account for all the evidence. Failing that, such an account would be so all encompassing as to be irrefutable. Take, for example, the reports of heightened response perseverative behaviors by rats with hippocampal damage. Many such observations could be broadly interpreted as illustrating the inability of the animal to tune out stimuli that control modes of behavior that are no longer appropriate. Such an interpretation might be compelling when applied to some cases of 
perseverative behaviors, but unconvincing in others. To illustrate, Franchina and Brown (1971) reported that hippocampectomized rats, unlike the controls, did not alter their speed of running in an alley way when reward magnitude is either increased or decreased. They continued to run at a speed appropriate to their initial experience in the apparatussignificantly faster if running to the larger of two reward magnitudes. When shifted to the smaller reward hippocampectomized rats did not slow down, nor did they speed up when shifted to the larger reward. This is an instance of response perseveration that I cannot fit into the tuning-out framework with conviction. On the other hand, I also find it difficult to reconcile this particular observation with the spatial processing hypothesis of Black et al. (1977). The point is that it is unlikely that either the spatial mapping or the tuning-out hypothesis, narrowly interpreted, can alone do justice to the relevant behavioral literature. One purpose of the present paper is to initiate a process of rigorous behavioral analysis of the behavioral literature relevant to the spatial mapping theory and see how this literature might be interpreted in terms of temporal processing, especially that part of temporal processing in which irrelevant events or associations need tuning out in order for efficient learning or performance to occur. ${ }^{3}$

\section{Spatial Mapping and Avoidance Learning}

Black et al. (1977) showed how the large and seemingly contradictory literature concerning the role of the hippocampus in avoidance learning might be understood in terms of the simple notion that animals with hippocampal damage have difficulty processing information about the places they encounter while learning to avoid shock. With impaired place learning, such animals must rely on "cue strategies." Cue learning, unlike place learning or spatial mapping, presumably does not involve the hippocampus. In some kinds of avoidance tasks, for example, two-way shuttle avoidance, the inability to employ place strategies actually results in improved performance. In other passive avoidance or punishment situations, appropriate behavior is impaired. In both cases, animals with hippocampal damage tend to be less reluctant than are controls to go to places where shock has been administered in the past.

According to Black et al. (1977), animals with hippocampal damage either do not possess or cannot make proper use of "cognitive maps" which represent the spatial layout of their worlds. The hippocampus is thought to be responsible for creating and storing cognitive maps that contain information about the spatial relationships among places. The integrity of a map is invariant under transformations of the animal's own position and orientation in space. Rearrangement of the location of stimulus objects does not change the map, except in the sense that the animal can take note of the rearrangement and pinpoint each stimulus object in terms of the map's coordinate system. Hippocampal damage is tantamount to destruction of the map, and the animal expresses this loss by behaving as though it cannot remember places. It can find its way about only with the aid of a beacon or cue.

Supporting the spatial mapping viewpoint is evidence that animals with hippocampal damage are impaired in tasks, such as elevated mazes with multiple starting points, which emphasize place as opposed to response strategies. Much of this evidence might be subsumed under the tuning-out view of hippocampal function, since it could be argued that it is precisely the inability to suppress an irrelevant strategy, e.g., that of making a previously rewarded response, that makes a place learning task so difficult for the animal with this type of brain damage (Herrmann, Black, Anchel, \& Ellen, 1978; Olton, 1972; except see Olton \& Werz, 1978).

I can perhaps illustrate this point by considering the eight-arm radial maze task employed by Olton and his associates (e.g., Olton \& Samuelson, 1976) to investigate spatial memory in rats. Beginning each trial from the hub at the center of the maze, the animal's task is to obtain reward from the baited goal area at the end of each arm or spoke. To do this within the allotted time, the animal must avoid reentering arms of the maze that it has already visited. This is a task that depends on spatial processing, and successful performance is based primarily on visual rather than olfactory cues (Zoladek \& Roberts, 1978).

Normal rats are remarkably proficient at this task, and their performance is not hindered very much by the imposition of a forced delay of up to $2 \mathrm{~min}$ half way through a trial or by a variety of interposed procedures designed to interfere with memory processes. For example, in one recent study "variations in visual and auditory environments, removal from the maze, or a combination of these manipulations" were all ineffective in disrupting spatial memory (Maki, Borkofsky, \& Berg, 1979, p. 25).

In short, appropriate behavior in the eight-arm maze is possible under circumstances in which most temporal-processing viewpoints might predict disruptions in performance. For this reason, the deficits in performing this task following hippocampal damage (Jarrard, 1978; Olton, 1977; Olton, Walker, \& Gage, 1978) only serve to emphasize the importance of these structures in learning and remembering the spatial rather than temporal aspects of the task.

An interpretation of lesion-produced deficits in spatial memory in terms of the tuning-out hypothesis would be that animals are able to perform this task only by subordinating associations between spatial 
cues and reward that would otherwise lead to reentry errors. Hippocampal rats would have difficulty doing this. Unlike failure to suppress inappropriate or obsolete response strategies (e.g., Olton, 1972), performance on the eight-arm radial maze may be especially susceptible to failure to suppress inappropriate strategies involving visual cues that control entry to arms of the maze. I am using the phrase "suppress inappropriate behavior strategies" to be roughly synonymous with the phrase "tune out stimuli that control inappropriate behavior." Seen in this light, the tuning-out viewpoint and spatial mapping viewpoint appear to be compatible.

The essential point is that although hippocampal damage can disrupt performance in spatial memory tasks, the psychological processes underlying such disruption may have a significant temporal dimension. This temporal dimension probably involves some form of interference, proactive, concurrent, or retroactive, such that irrelevant associations compete with appropriate behavior in the task at hand. In the eight-arm maze, the hippocampal animal is more prone to making reentry errors. This suggests proactive interference. The same source of interference has been cited by Sinnamon, Freniere, and Kootz (1978) to account for their finding that rats with fornix lesions or bilateral lesions of the dorsal hippocampus are unable to recall the place where they had encountered reward just $15 \mathrm{sec}$ previously. These authors stress the likelihood that encoding processes were affected by the lesions and that proactive interference was responsible for the memory deficits.

In addition to evidence that hippocampally damaged animals are impaired in some spatial tasks, hippocampal damage has been shown to potentiate the effects of interference in such tasks. For example, Jarrard (1975) reported on the interference produced by interpolated activity in a task in which rats were rewarded for alternating choices in a Y-maze. Forced wheel running in the startbox area during an intertrial delay produced more interference in hippocampal rats than in control animals. Since the animals were allowed to enter the startbox as soon as a bout of forced running came to an end, it is possible that running became a signal for reward. Unless the animal ignored or tuned out this irrelevant association, it is likely that indiscriminate running would interfere with other behaviors, such as VTE-ing at the choice point, which would be the more appropriate strategy in this task. In the normal rat, the interference from the irrelevant association of running with reward would be blocked by associative connections between spatial cues and/or choice point behavior and the reward. These associations would have precedence through learning prior to the introduction of interpolated running. The irrelevant association between running per se and reward would not be blocked so readily in rats with hippocampal damage, any more than Solomon's (1977) rabbits were able to prevent developing a conditioned response to the redundant element of a compound $\mathrm{CS}$ in which the other element had been previously conditioned.

The foregoing analysis of potentiated interference resulting from hippocampal damage in Jarrard's (1975) study rests on an assumption that blockinglike processes can occur not only with respect to external cues such as Pavlovian conditioned stimuli, but also with respect to the cuing function of the animal's own behavior or behavioral state. Although the validity of this assumption is yet not well documented, animal learning theorists (e.g., Mackintosh \& Dickinson, 1979) are gathering relevant evidence.

The analysis also assumes that if event $A$ precedes event $B$ in time, but $B$ had been associated with reinforcement before the introduction of $A$, then $B$ would block the association of $\mathrm{A}$ with the reinforcer despite the fact that $\mathrm{A}$ is the initial event in the sequence. A recent conditioned-suppression study has shown that prior conditioning to $\mathrm{B}$ can block conditioning to $\mathrm{A}$ when $\mathrm{A}$ and $\mathrm{B}$ are sequentially presented conditioned stimuli (Kohler, 1979). This finding parallels my analysis of interference in Jarrard's (1975) study in that choice-point cues and behaviors (B) were associated with reward prior to the introduction of forced running (A). These associations presumably block the development of an association between running and reward in the normal animal and thereby mitigate this source of interference. Hippocampal animals, less able to block irrelevant or redundant associations with reward, show impaired performance on the original task.

\section{Selectivity of Effects of Hippocampal Damage}

Proponents of the spatial mapping view could challenge my interpretation of spatial learning deficits by raising the question of why hippocampal damage selectively disrupts place but not response learning. More to the point, why would not the perseveration of a rewarded response disrupt cue learning in the same way it disrupts place learning? The best way to answer this is to consider the evidence bearing on selectivity and dissociation of function in detail. A recent report by Olton, Branch, and Best (1978), proponents of the spatial mapping viewpoint, cites two studies relevant to the question of selectivity, Mahut (1972) and O'Keefe, Nadel, Keightly, and Kill (1975), and they are considered in the proper place later in this article. The largest body of data supporting selectivity of hippocampal function are the experiments on avoidance and punishment reviewed by Black et al. (1977). Black et al. (1977) also cite taste-aversion learning studies in support of the spatial mapping theory, but as 
Solomon (1979) has shown, failure to disrupt simple classical conditioning does not imply that the hippocampus is uninvolved in phenomena closely related to classical conditioning. For example, McFarland et al. (1978) report deficits of latent inhibition in taste-aversion learning in rats analogous to those reported in rabbits by Solomon and Moore (1975).

Black et al. (1977) ascribe the widely reported finding of facilitated two-way avoidance in animals with hippocampal damage to the inability of these animals to pinpoint the location of "dangerous places" in their spatial map. Guided solely by nonspatial cues, they are not reluctant to enter a compartment where they had previously experienced shock. The recall of a to-be-encountered dangerous place does not arise in one-way avoidance, and the weight of evidence supports the idea that animals with hippocampal damage do not differ from normals in this type of task. Similar considerations can account for the fact that punishment following locomotion is less effective in slowing animals with hippocampal damage than it is in slowing controls. In short, where the need to recall the location of a dangerous place does not arise, hippocampals and normals do not differ.

At least implicitly, Black et al. (1977) assume that animals with hippocampal damage are able to associate place cues with shock. It is disruption of the spatial mapping systems which precludes their acting on the basis of this information. An alternative account is that, under certain circumstances, animals with hippocampal damage have difficulty associating place cues with shock and that the failure of association is at least partly responsible for the pattern of evidence reviewed by Black et al. (1977) in support of their position. The basis of this failure of association may be found in the inability of animals with hippocampal damage to tune out unexpected or incongruent information-information that may be particularly disruptive to new learning. Details of an experiment pointing toward this possibility and the possible bearing on avoidance and punishment are presented later in this article.

First, I wish to consider the two studies cited by Olton et al. (1978) as providing especially convincing support for the notion that hippocampal damage selectively disrupts spatial learning while leaving cue learning intact. Without questioning the selectivity of the effects reported in these studies, I believe they can be reconciled with the tuning-out hypothesis.

Spatial vs. object reversal learning in monkeys. Mahut (1972) trained macaque monkeys with transected fornices and other lesions of the hippocampal complex on a series of visual discrimination problems. Using a modified Wisconsin General Test Apparatus (WGTA) in which an opaque screen separated the animal from the display tray, performance of these animals was compared with that of normal monkeys and monkeys with control operations. The problems of special interest were spatial reversals and object reversals. The basic procedure is described as follows:

Spatial reverals. Two identical gray plaques were used and, for all animals, the bait was on the left side during initial learning. Using a non-correction procedure, thirty trials a day were given until a learning criterion of 18 correct responses in 20 consecutive trials was reached. This was followed, within the same session, by a reversal to the opposite side, for another 20 trials . . . Successive reversals were repeated until the monkeys reversed within a session with no more than two errors in 20 trials or to a maximum of 19 reversals . . . .

Object reversals. The procedure was exactly the same as. that followed for spatial reversals but this time two three-dimensional junk objects were used (a plastic red tomato vs. three large paper clips) and, while their position varied in a predetermined order between two locations, monkeys had to choose a previously incorrect object on each consecutive reversal [Mahut, 1972, p. 68].

Experimental and control monkeys did not differ significantly on either initial position learning or on initial object learning. Nor did the two groups differ on object reversals, experimental animals performing just as efficiently as controls. On position reversals, however, the experimental animals were significantly impaired compared to controls. How can we account for this selectivity of impairment related to fornical and, in some cases, other hippocampal damage?

I believe the explanation can be found in different observing behaviors instilled in the course of learning the two types of problems. Monkeys look where their fingers go. In the WGTA, they tend to limit their observing behavior to stimuli which keep them on track toward the goal, and separating the relevant cue from the locus of reward disrupts the ability of intact monkeys to solve discrimination problems (cf. Stollnitz, 1965).

In position learning, monkeys tend to look where the bait has consistently been, and it is likely that reaching-looking begins as soon as the screen starts to lift. When reversals begin, the animal must shift or redirect its observing response to the new position. Monkeys with fornix lesions may have difficulty doing this. Such animals might continue to look toward the wrong place and to persist in responding to stimuli no longer relevant to the problem. An intact hippocampus enables a monkey to eventually focus on relevant stimuli by tuning out the irrelevant ones that no longer lead to reward.

The same analysis can be extended to an experiment by Mahut and Zola (1973) in which fornixlesioned and control monkeys were compared on spatial and object reversals, but where the problems 
were performed in infrared light on the basis of tactile cues. The results were basically similar to those obtained by Mahut (1972) in the earlier study. In object discrimination learning, monkeys learned to touch both objects in order to locate the position on the display tray of the correct one. Such observing behavior continues to be appropriate following reversal, and as a consequence, monkeys with fornix lesions performed just as efficiently as controls. In initial position learning, monkeys probably learned to grope in one direction and experimental monkeys may have had difficulty tuning out whatever stimuli controlled this behavior when it was no longer appropriate.

The above accounts contain unstated assumptions and have been embellished with details for which no evidence has been presented-for example, the notion that a monkey's hand moves toward the correct place before the test stimuli come fully into view. Evidence of such behavior would strengthen my contention that monkeys employ different observational strategies in solving the two types of discrimination problems considered here. Some support for this contention can be drawn from a recent study of recognition memory for colors and spatial location in fornix-transected monkeys. Gaffan (1977) employed a panel-pushing task that ensured that the animal observed the to-be-recognized stimuli. There was no evidence of selectivity in this study, as fornix-transected monkeys, though impaired in comparison with controls, were no worse on the spatial task than on the color task.

Proponents of the spatial mapping viewpoint would likely argue that the fornix-lesioned monkeys in Mahut's studies had impaired spatial mapping systems. I attribute the selectivity of impairment to the selective action of the hippocampal system in tuning out irrelevant stimuli. Clearly, not all irrelevant stimuli produce learning deficits. Whether attention to irrelevant stimuli adversely affects an animal's ability to attend to relevant cues depends on whether the two processes are in conflict.

Place vs. cue learning. O'Keefe et al. (1975) compared rats with fornix lesions with operated controls in an experiment designed to demonstrate selective abolition of place learning by the lesion. In this innovative and carefully thought out study, groups of rats from each surgical treatment were trained to locate water using either a cue or place strategy. There were four groups in all, representing the various combinations of surgical treatment and type of learning. All groups were trained and tested on the same apparatus, a circular elevated runway consisting of eight equally spaced watering holes or wells, one located every $45^{\circ}$, imbedded in the running surface.

The apparatus was located in a room with many distinctive place cues, including the experimenter who always sat in the same place some $3 \mathrm{~m}$ from the runway. A trial was initiated by placing the animal at a point on the runway, chosen at random and varied from trial to trial, and allowing 2 min for it to locate the well from which water would be available. For cue learners, the correct well was illuminated by an $8-\mathrm{cm}$ spotlight, the actual location varying from trial to trial. Place learners also experienced the light, the light and water occurring together one trial in eight on the average. Place learners were always rewarded at the same place, varied from rat to rat, and the spotlight was an irrelevant cue. The runway was rotated from trial to trial in order to control for intramaze cues, such that, for place learners, the correct well was always in the same place with respect to extramaze cues. Care was taken to control for learning based on olfaction. On each trial of training and testing, if 2 min elapsed without success the animal was gently pushed to the correct water hole and allowed to drink.

With these procedures, all rats with fornix lesions in the place-learning group went through the entire 55 trials of testing ( 5 trials per day following 25 pretraining trials) without once finding the correct water hole in the time permitted. Fornix-lesioned rats in the cue learning group and both control groups attained a criterion of 9 correct trials in 10 in less than 10 trials, on the average. Winson (1978) has reported a similar impairment in this circular maze task in rats given theta-disrupting lesions of the medial septal area. Lesions of the fornix or medial septum clearly affected learning in a selective fashion, producing a substantial deficit in place learning but no deficit in cue learning. The question is, how come?

O'Keefe et al. (1975) interpret their data to indicate that cue learners employed a cue strategy that consisted in looking for the spotlight and locomoting toward it. Given this simple strategy of sign tracking (Hearst \& Jenkins, 1974), control and fornix-lesioned rats performed with no difficulty. Nor would we have expected them to. The place learners unfortunately must learn that the cue light is irrelevant to locating water despite its close association with reward on some trials.

The place learner must associate the reward with its position in relation to extramaze cues. In Pavlovian language, the correct place can be viewed as a stimulus that is paired with reinforcement $100 \%$ of the time, but sometimes the cue light was part of this stimulus complex. While blocking would normally prevent development of an association between the cue light and drinking, because the cue light is part of the complex only a portion of the time, place learners with fornix lesions might have been unable to tune out this redundant and irrelevant cue. Failure to block conditioning to the cue light could have resulted in rats with fornix lesions being drawn by 
the cue light to incorrect places. This argument assumes that place learners do not learn that the cue light is more often associated with nonreward than with reward. This may be an error, but then so might the assumption implicit in O'Keefe et al.'s (1975) experiment that place learners always notice the cue light and can therefore learn its true correlation with reward.

Another unwanted association, one which may have been the more likely culprit in abolishing place learning in rats with fornix lesions, is that between the water holes and reward. Viewed proximally, all the wells were indistinguishable from each other. During testing, the animals were required to lick the correct well for water to be delivered, and all trials ended with reinforcement even if this necessitated pushing the rat to the correct well. Control rats presumably learned to ignore the proximal aspects of water holes as being irrelevant to locating reward, but rats with fornix lesions became increasingly drawn into an inefficient pattern of responding. Consider the description of the behavior of such rats: "Most ... animals ran around the maze on the first few trials, licking none of the wells, or at most one or two incorrect ones. When this strategy failed, they developed a new strategy of licking at all the wells between the start segment and the correct well, a strategy they maintained throughout the study", (O'Keefe et al., 1975, p. 158).

Instead of indicating a shift of strategies, this account can be interpreted just as readily in terms of sign tracking. Early difficulties encountered in learning the location of reward on the basis of place could have conspired with partial reinforcement effects to produce a very robust approach response.

Summarizing, both groups of cue learners in the O'Keefe et al. (1975) study needed only to approach the cue light in order to obtain reward. Place learners were taught to approach water holes. This proved to be inappropriate behavior, and the place learners had to learn to ignore the proximal cues provided by water holes and shift their observing responses to other stimuli. Fornix-lesioned rats, like Mahut's fornix-lesioned monkeys, would have difficulty making this shift (see also Olton, 1972). Such rats might, indeed, have impaired spatial mapping systems, but it seems equally plausible to suppose they are unable to tune out inefficient modes of responding.

\section{Going to Shocking Places: \\ Failures of Association?}

Black et al. (1977) maintain that animals in avoidance and punishment tasks employ both place and response strategies. Hippocampal damage precludes utilization of the former because of disruption of the spatial mapping system. Accordingly, animals with hippocampal damage are faster to acquire a signaled two-way avoidance response because, unlike normals, they cannot be in conflict about returning to places they have experienced shock. Animals with hippocampal damage are relatively impaired in situations where they must go from one place to another and are punished when they arrive, i.e., they are less hesitant than normals to locomote toward a dangerous place. Unable to employ a place strategy, they must resort to less efficient cue strategies in order to suppress punished behavior.

In order to employ a place strategy, the animal needs to be able to form the associative network that constitutes the relevant spatial map. I suggest that animals with hippocampal damage have difficulty forming such an associative network ("encoding problem") because they may have difficulty tuning out a class of irrelevant events that appear to be particularly important in associative learning. This class of events has been dubbed incongruent by Wagner, Rudy, and Whitlow (1973). Incongruence is defined as evidence of an altered associative relationship involving the reinforcer. Wagner et al. (1973) presented rabbits undergoing eyeblink conditioning with one of two kinds of events following reinforcement of the target CS. One event was congruent with prior learning in that it did not imply a change in a previously experienced reinforcement contingency; the other event was incongruent because it did. Rabbits presented the incongruent stimulus following a trial were slower to condition to the target CS than were those presented a congruent or neutral posttrial event. Wagner et al. (1973) took this evidence to mean that the animal's rehearsal of the target association was prematurely cut short by rehearsal of the incongruent information. Clearly, incongruent information is more disrupting than are such things as novel stimuli or distractors. All else being equal, incongruent events evidently evoke more prolonged bouts of rehearsal at the expense of time that would otherwise be devoted to the target association than do other kinds of events.

The incongruous feature of two-way avoidance is that of requiring the animal to shuttle from a place where it has just experienced shock to another, typically similar, place where shock is also presented, but only after an "intertrial interval" and provided the animal does not successfully avoid. The animal must learn where each place is in relation to the other and that each place is dangerous. This might be too much to expect of an animal with hippocampal damage faced with the incongruity of learning that it is its own behavior that controls the shock and not the conflicting signals from its environment. This incongruity, i.e., inconsistency regarding the relative danger or safety of places, might also be expected to disrupt the cue learning aspect of shuttle avoidance. 
Perhaps it does to a degree, but not enough to offset the net advantage provided by associative failures related to places.

In punishment situations, shock is typically presented at a point in the animal's experience when the associative network defining the location of various places is intact. Nevertheless, the unexpected introduction of shock could disrupt fragile but important associative links, for example, remote associations between place cues in the start compartment and in areas leading up to the place where shock is delivered. Startbox and runway latency are good indicators of these associations, and it is perhaps not surprising that these are the measures of punishment most likely to show a deficit as a result of hippocampal damage (see Black et al., 1977; Table 6, p. 1120).

In sum, I am suggesting that time spent processing or rehearsing unexpected events can interfere with the formation of associations between place cues and shock. When the separation is relatively great in terms of distance and time, the possibility of interference may be greater than when the two occur close together (Revusky, 1971). Unlike animals with hippocampal damage, normal animals may be able to ignore the unexpected or incongruous and in this way establish remote associations between places and shock free from interference.

\section{Information Processing in Space-Time}

As the nature of information processing underlying spatial learning becomes an issue of concern, it becomes apparent that time is a relevant aspect of the problem. The involvement of the hippocampus in this domain may be most easily described as one of tuning out those irrelevant, but otherwise salient, events that intrude on associative learning or that lead to inefficient modes of responding. Determination of precisely what these events might be requires approaching each example of spatial learning with an open mind and from a fresh viewpoint.

The spatial map formed as an animal explores its environment contains associative links not only between places, but between each place and itself as it moves in space and time. The animal encounters the things in its environment sequentially, scanning various places near or far in turn, forming and perhaps breaking associations along the way as a cognitive map evolves. The resulting associative network probably contains not only knowledge of places, but also information about the elapsed time separating one place from another. Time and space become inextricably entwined.

As Solomon (1979) notes, the spatial mapping viewpoint seems most plausible when one is studying an animal's behavior in relatively unconfined spaces. A temporal mapping viewpoint seems most attractive in situations where space is limited. In each case, the investigator tends to view the relevant cognitive map on edge, i.e., with a key dimension masked by the nature of the task. For example, it is only in the highly controlled and restricted context of classical conditioning that the spatial mapping process becomes irrelevant. Yet the converse does not apply when considering an animal's experiences with places or other salient features of its environment.

Solomon (1979) and I have argued that the hippocampus is not solely concerned with spatial learning, but that the selective character of behavioral deficits can be interpreted in terms of a general informationprocessing function of the hippocampus. Instead of regarding the hippocampus as the repository of spatial engrams or cognitive maps, it may be more useful to regard the function of the hippocampus as one of tuning out irrelevant stimuli or events. Such events could conceivably be represented as points on a cognitive map of space-time. It may be in this role that the relationship of the hippocampus to spatial learning can be best understood.

At this juncture, the available evidence indicates that the hippocampus participates in both spatial and temporal mapping. If this is so, the hippocampus might be regarded as part of a system that constructs or permits access to cognitive maps which include time as one of their dimensions. Such a synthesis may be possible, and whatever contradictions remain between the spatial mapping hypothesis and the tuningout hypothesis might be reconciled in this way.

\section{REFERENCES}

Berry, S. D., \& Thompson, R. F. Medial septal lesions retard classical conditioning of the nictitating membrane response in rabbits. Science, 1979, 205, 209-211.

BlaCk, A. H., NADEL, L., \& O'KeEFe, J. Hippocampal function in avoidance learning and punishment. Psychological Bulletin, 1977, 84, 1107-1129.

Douglas, R. J. Pavlovian conditioning and the brain. In R. A. Boakes \& M. S. Halliday (Eds.), Inhibition and learning, New York: Academic Press, 1972.

Franchina, J. J., \& Brown, T. S. Reward magnitude shift effects in rats with hippocampal lesions. Journal of Comparative and Physiological Psychology, 1971, 76, 365-371.

GAFFAN, D. Behavioral contrast and inhibitory gradients in rats with lesions of the fornix. Physiology \& Behavior, 1973, 11, 215-220.

GAFFAN, D. Recognition memory after short retention intervals in fornix-transected monkeys. Quarterly Journal of Experimental Psychology, 1977, 29, 577-588.

Hearst, E., \& Jenkins, H. M. Sign-tracking: The stimulusreinforcer relation and directed action. Austin, Texas: Psychonomic Society, 1974.

Herrmann, T., Black, A. H., Anchel, H., \& Ellen, P. Comparison of septal and fornical lesioned rats' performance on the Maier three table reasoning task. Physiology \& Behavior, 1978, 20, 297-302.

JARRARD, L. E. Role of interference in retention by rats with hippocampal lesions. Journal of Comparative and Physiological Psychology, 1975, 89, 400-408. 
JARRARD, L. E. Selective hippocampal lesions: Differential effects on performance by rats of a spatial task with preoperative versus postoperative training. Journal of Comparative and Physiological Psychology, 1978, 92, 1119-1127.

KoHLER, E. A. Serial compounds and trace conditioning procedures in the Kamin blocking paradigm. Unpublished doctoral dissertation, University of Massachusetts, Amherst, 1979.

Mackintosh, N. J. A theory of attention: Variations in the associability of stimuli with reinforcement. Psychological Review, 1975, 82, 276-298.

Mackintosh, N. J., \& Dickinson, A. Instrumental (Type II) conditioning. In A. Dickinson \& R. A. Boakes (Eds.), Mechanisms of learning and motivation: A memorial volume to Jerzy Konorski. Hillsdale, N.J: Erlbaum, 1979.

MAнUT, H. A selective spatial deficit in monkeys after transection of the fornix. Neuropsychologia, 1972, 10, 65-74.

Mahut, H., \& ZolA, S. M. A non-modality specific impairment in spatial learning after fornix lesions in monkeys. Neuropsychologia, 1973, 11, 255-269.

MAKI, W. S., Brokofsky, S., \& Berg, B. Spatial memory in rats: Resistance to retroactive interference. Animal Learning \& Behavior, 1979, 7, 25-30.

McFarland, D. J., Kostas, J., \& Drew, W. G. Dorsal hippocampal lesions: Effects of preconditioning CS exposure on flavor aversion. Behavioral Biology, 1978, 22, 398-404.

Micco, D. J., \& Schwartz, M. Effects of hippocampal lesions upon the development of Pavlovian internal inhibition. Journal of Comparative and Physiological Psychology, 1971, 76, 371-377.

Moore, J. W. Brain processes and conditioning. In A. Dickinson \& R. A. Boakes (Eds.), Mechanisms of learning and motivation: A memorial volume to Jerzy Konorski. Hillsdale, N.J: Erlbaum, 1979.

O'Keefe, J., Nadel; L., Keightly, S., \& Kill, D. Fornix lesions selectively abolish place learning in the rat. Experimental Neurology, 1975, 48, 152-166.

Otton, D. S. Behavioral and neuroanatomical differentiation of response-shift and response suppression mechanisms in the rat. Journal of Comparative and Physiological Psychology, 1972, 78, 450-458.

Otton, D. S. Spatial memory. Scientific American, 1977, 236(b), 82-98.

Olton, D. S., Branch, M., \& Best, P. J. Spatial correlates of hippocampal unit activity. Experimental Neurology, 1978, 58, 387-409.

Olton, D. S., \& Samuelson, R. J. Remembrance of places passed: Spatial memory in rats. Journal of Experimental Psychology: Animal Behavior Processes, 1976, 2, 97-116.

Olton, D. S., Walker, J. A., \& Gage, F. H. Hippocampal connections and spatial discrimination. Brain Research, 1978, 139, 295-308.

Olton, D. S., \& Werz, M. A. Hippocampal function and behavior: Spatial discrimination and response inhibition. Physiology \& Behavior, 1978, 20, 597-605.

REVUSKY, $\mathrm{S}$. The role of interference in association over a delay. In W. K. Honig \& H. James (Eds.), Animal memory. New York: Academic Press, 1971.
Rickert, E. J., Bennett, T. L., Lane, P., \& French, J. Hippocampectomy and the attenuation of blocking. Behavioral Biology, 1978, 22, 147-160.

Salafia, W. R., Romano, A. G., Tynan, T. T., \& Host, K. C. Disruption of rabbit (Oryctolagus cuniculus) nictitating membrane conditioning by post-trial electrical stimulation of hippocampus. Physiology \& Behavior, 1977, 18, 207-212.

Sinnamon, H. M. Freniere, S., \& Kootz, J. Rat hippocampus and memory for places of changing significance. Journal of Comparative and Physiological Psychology, 1978, 92, 142-155.

Solomon, P. R. Role of the hippocampus in blocking and conditioned inhibition of the rabbits' nictitating membrane response. Journal of Comparative and Physiological Psychology, 1977, 91, 407-417.

Solomon, P. R. Temporal versus spatial information processing theories of hippocampal function. Physiological Bulletin, 1979, in press.

Solomon, P. R., \& Moore, J. W. Latent inhibition and stimulus generalization of the classically conditioned nictitating membrane response in rabbits (Oryctolagus cuniculus) following dorsal hippocampal ablation. Journal of Comparative and Physiological Psychology, 1975, 89, 1192-1203.

STollnitz, F. Spatial variables, observing responses, and discrimination learning sets. Psychological Review, 1965, 72, 247-261.

Wagner, A. R., Rudy, J. W., \& Whitlow, J. W. Rehearsal in animal conditioning. Journal of Experimental Psychology, 1973, 97, 407-426. (Monograph)

WEISKRANTZ, L., \& W ARRINGTON, E. K. The problem of amnesic syndrome in man and animals. In R. L. Isaacson \& $\mathrm{K}$. H. Pribram (Eds.), The hippocampus (Vol. 2). New York: Plenum Press, 1975.

Winson, J. Loss of hippocampal theta rhythm results in spatial memory deficit in the rat. Science, 1978, 201, 160-163.

ZolaDeK, L., \& RoBerts, W. A. The sensory basis of spatial memory in the rat. Animal Learning \& Behavior, 1978, 6, 77-81.

\section{NOTES}

1. Disruptions of hippocampal function by electrical stimulation (Salafia, Romano, Tynan, \& Host, 1977) or selective deafferention (Berry \& Thompson, 1979) can retard conditioning of the rabbit's nictitating membrane response.

2. Analogous experiments with rats have yielded mixed results, some evidence suggesting impaired inhibitory learning (Micco \& Schwartz, 1971) and other evidence suggesting that inhibition is unaffected by hippocampal-related lesions (e.g., Gaffan, 1973). The difficulty with these studies is one of ascertaining whether the presumed inhibitory stimuli possess all the properties of true conditioned inhibitors (see Moore, 1979, and Solomon, 1977, for discussion of this point)

3. The reader is referred to Solomon (1979) for a review of electrophysiological studies relevant to spatial and temporal processing by the hippocampus.

(Received for publication October 23, 1978; revision accepted June 27,1979 .) 